\title{
Surgery for cervical myelopathy in geriatric patients
}

\author{
N Razack ${ }^{1}, \mathrm{~J}_{\text {Greenberg }}^{2}$ and BA Green ${ }^{1}$ \\ ${ }^{1}$ Department of Neurological Surgery, University of Miami School of Medicine and ${ }^{2}$ Orlando Regional Medical \\ Center, Florida, USA
}

\begin{abstract}
Objective The number of geriatric patients seeking surgical treatment for cervical myelopathy is steadily increasing. Although anecdotal experiences have been good, insufficient data exists in the spine literature concerning this particular group. We decided to review our experience to determine efficacy of surgical management and examine our morbidity with this select group. Methods We undertook a retrospective review of all surgical procedures for nontraumatic cervical myelopathy performed at Mt Sinai Medical Center and Jackson Memorial Medical Center between January 11987 and June 1 1992, in patients older than 70 years of age (33 men and 18 women).

Results A total of 53 cervical surgical procedures were performed in 51 patients (nine expansile laminoplasties, 20 anterior cervical diskectomies and fusion and 24 decompressive posterior laminectomies). The average hospital stay was $7.7 \pm 3.4$ days. Twenty-one (41\%) patients required inpatient rehabilitation; the remaining 30 patients received outpatient rehabilitative therapy. The major morbidity rate was $3.9 \%$, and the minor morbidity rate was $5.8 \%$. Perioperative medical complications included cardiac arrhythmias, hypertensive episodes, atelectasis, confusion, urinary dysfunction and hyponatremia. All of these complications resolved except in one patient. The perioperative mortality rate was $2 \%$. Office follow-up was performed for a mean of $11.1 \pm 2.5$ months postoperatively. Comparison of preoperative and postoperative functional status was performed using Nurick's criteria as described in the literature. At follow-up, $60.8 \%$ of the patients had improvement in their myelopathic symptoms, especially in regard to gait; $33.3 \%$ were stabilized and $5.9 \%$ had worsened neurological function.

Conclusion This study demonstrates that corrective surgical procedures for significant nontraumatic cervical myelopathy in the geriatric population may be performed safely, that is, with acceptable risk of morbidity and reasonable expectation for clinical improvement.
\end{abstract}

Keywords: cervical spondylitic myelopathy; geriatric; Nurick's criteria; surgery

\section{Introduction}

Geriatric patients are now commonly seeking neurosurgical treatment for cervical myelopathy. Two factors directly related to this phenomenon are the increase in size of the geriatric population and the availability of such noninvasive neuroradiological imaging modalities as magnetic resonance imaging for the evaluation of cervical myelopathy. Some surgeons are rightly concerned about the increased risk of medical and surgical complications associated with undertaking major spinal corrective procedures in elderly patients. Although many spine surgeons anecdotally state they have experienced no increased morbidity in elderly patients there is insufficient data to guide the surgeon in estimating risk in such cases. Many internists remain skeptical of referring elderly patients to undergo major spinal corrective procedures because of fear of increased medical or surgical complications. Previous

Correspondence: Dr J Greenberg, Department of Neurological Surgery, Orlando Regional Medical Center, Orlando, Florida, 32806, USA reports on cervical spine surgery for cervical spondylitic myelopathy (CSM) have been procedure specific and have not analyzed older patients as a separate group. ${ }^{1-6}$ There are very few population-specific studies of CSM and its surgical management. The purpose of this review was to determine the efficacy of surgical management of CSM in geriatric patients and to assess the perioperative medical complications most likely to be encountered in this population.

\section{Patients and methods}

We undertook a retrospective review of all surgical procedures for nontraumatic CSM in patients older than 70 years of age at Mt Sinai Medical Center, Miami Beach, Florida and Jackson Memorial Hospital, Miami, Florida, between January 11987 and June 1 1992. The patients' medical records were reviewed with regard to presenting neurological symptoms, preoperative medical problems, type of surgical procedure performed, medical and surgical complications, length 
of hospitalization and ultimate disposition. The operative procedures were not chosen in a randomized fashion. Therefore, we are not making any comparisons for the choice of surgical techniques nor are we commenting on the criteria to make that decision. Anatomic diagnosis of spondylitic spinal cord composition was confirmed with cervical spine radiographs, computed tomography-myelography or magnetic resonance imaging. In some patients, more than one imaging modality was utilized. American Society of Anesthesiologists (ASA) grades were assessed preoperatively. Preoperative and postoperative functional status was assessed using Nurick's criteria ${ }^{12,13}$ as modified by Lesoin et al. (Table 1). Statistical analysis comparing the preoperative and postoperative Nurick stage was performed using repeated measures of analysis of variance. Although other criteria for assessing functional status are reported in the literature, ${ }^{10,11}$ Nurick's criteria were found to be the easiest to apply, and because of their simplicity, the most likely to minimize observer bias. Whenever possible, physical therapy assessment forms were used to augment physician assessment. The average followup period for patients was $11.1 \pm 2.5$ months after surgery. All patients were placed in either a Miami-J cervical collar or a Philadelphia collar for a period of $8-10$ weeks postoperatively. Follow-up flexion/extension radiographs were then obtained at that time.

\section{Results}

Fifty-three cervical procedures were performed in 51 patients (33 men and 18 women). The average age was $73.1 \pm 1-5.12$ years. The duration of symptoms of myelopathy ranged from 1 month to 10 years (mean 1.2 years). Gait ataxia, with or without loss of fine finger control was the most frequent primary clinical presentation (30/51), followed by chronic motor/ sensory signs (12/51) and neck pain (9/51).

The premorbid medical conditions present at the time of admission are listed in Table 2. Systemic hypertension (19/15) and heart disease $(15 / 51)$ were the most frequently encountered conditions. The preoperative American Society of Anesthesiologists grades of physical status ranged from 2-4.

The following surgical procedures were performed: nine expansile laminoplasties, 20 anterior cervical diskectomies and fusion (with or without corpectomy or osteosynthetic plating) and 24 decompressive

Table 1 Nurick's criteria as described by Lesoin et al

\begin{tabular}{ll}
\hline Stage I & $\begin{array}{l}\text { Normal activities of daily living without func- } \\
\text { tional difficulty }\end{array}$ \\
Stage II & $\begin{array}{l}\text { Slight functional difficulty, virtually normal } \\
\text { activities of daily living }\end{array}$ \\
Stage III & $\begin{array}{l}\text { More marked difficulty requiring a little help } \\
\text { Considerable difficulty requiring aid of a person }\end{array}$ \\
Stage IV & $\begin{array}{l}\text { Bedridden } \\
\text { Stage V }\end{array}$
\end{tabular}

posterior laminectomies. Two patients $(3.9 \%)$ required repeat surgical procedures (Table 3). The average hospital stay was $7.7 \pm 3.4$ days. Twenty-one $(41 \%)$ patients required inpatient postoperative rehabilitation; the remaining 30 patients (59\%) received outpatient rehabilitative therapy. There was one death from cardiac arrest of unknown etiology (mortality rate $=2 \%$ ). Major surgical morbidity included one case of worsening neurological deficit, for which no etiology was found other than progression of myelopathy, and one case of wound dehiscence. There were two reoperations, one for inadequate bone removal and one for loosening of a cervical osteosynthetic plate.

Minor surgical morbidity included two cerebrospinal fluid leaks that resolved following lumbar drain placement and one wound seroma that resolved spontaneously. Perioperative medical complications included cardiac arrhythmias, transient hypertensive episodes, atelectasis, pulmonary edema, episodes of confusion and urinary tract infection (Table 4). Significant medical complications causing increased length of hospital stay was found in $5 / 51(9.8 \%)$ of the patients.

The mean preoperative and postoperative Nurick stages for the 51 patients were $2.33 \pm 0.62$ and

Table 2 Premorbid medical conditions among elderly patients with cervical spondylitic myelopathy

\begin{tabular}{lc}
$\begin{array}{l}\text { Premorbid medical } \\
\text { condition }\end{array}$ & $\begin{array}{c}\text { No. of Patients } \\
(\mathrm{n}=51)\end{array}$ \\
\hline Hypertension & 19 \\
Heart disease & 15 \\
Diabetes mellitus & 8 \\
Pulmonary disease & 5 \\
Thyroid disease & 3 \\
Depression/dementia/psychosis & 5 \\
Prostatic & 9 \\
Peptic ulcer disease & 3 \\
Peripheral vascular disease & 2 \\
\hline
\end{tabular}

Table 3 Surgical procedures performed among elderly patients with cervical spondylitic myelopathy*

\begin{tabular}{ll}
\hline Surgical procedures performed & $\begin{array}{c}\text { No. of procedures } \\
(\mathrm{n}=53)\end{array}$ \\
\hline $1 \quad$ Expansile laminoplasty & 9 \\
2 Anterior cervical diskectomy and & \\
fusion (with or without corpectomy & \\
or osteosynthetic plating) & 3 \\
$\quad$ One-level & 7 \\
$\quad$ Two-level & 6 \\
$\quad$ Three-level & 4 \\
$\quad$ Four-level & 24 \\
\hline
\end{tabular}

*Two patients each had two surgical procedures 
$1.72 \pm 0.91$, respectively, $(F=46.74 ; \mathrm{df}=1.50 ; P<0.01)$. At last follow-up, $60.8 \%$ of patients had improvement in their myelopathic symptoms, especially in regard to gait; $33.3 \%$ were stabilized, and $5.9 \%$ had worse neurologic function (Table 5).

\section{Discussion}

The results of surgical treatment of CSM must be evaluated within the context of the natural history of disorder. It is plausible that changes that occur in a patient's neurological status happen independently of any treatment. Clark and Robinson in 1956 published a study of 120 patients with CSM prior to any intervention. Clinical onset was most often accompanied by leg spasticity and weakness occurring during the fifth decade. Complete remission never occurred, and there was gradual clinical deterioration between episodes of new symptoms and signs. Nonoperative management improved CSM in Clark and Robinson's study.

Lees and Turner $^{10}$ in 1963 stated that CSM is a disease with a long clinical course characterized by periods of nonprogressive disability and rare occurrences of deterioration. Age of onset was not related to prognosis, which they found to be good without

Table 4 Medical complications among elderly patients with cervical spondylitic myelopathy

\begin{tabular}{lc}
\hline Complications & $\begin{array}{c}\text { No. of patients } \\
(\mathrm{n}=51)\end{array}$ \\
\hline Urinary tract infections & 1 \\
Cardiovascular arrest & 1 \\
Syncope & 1 \\
Hypertension & 5 \\
Arrhythmia & 3 \\
Confusion & 3 \\
Pulmonary edema & 1 \\
Atelectasis & 7 \\
Hyponatremia & 1 \\
\hline
\end{tabular}

Table 5 Preoperative Nurick stage and outcome of elderly patients with cervical spondylitic myelopathy

\begin{tabular}{ccl}
$\begin{array}{c}\text { Preoperative } \\
\text { Nurick stage }\end{array}$ & $\begin{array}{c}\text { No. of patients } \\
(\mathrm{n}=51)\end{array}$ & \multicolumn{1}{c}{ Outcome } \\
\hline I & 2 & $\begin{array}{l}\text { Stabilized, no change } \\
21 \text { improved to Stage I } \\
10 \text { stablilized to Stage II }\end{array}$ \\
II & 32 & 1 worsened to Stage III \\
& & 2 improved to Stage I \\
8 improved to Stage II \\
III
\end{tabular}

treatment. In contrast, Nurick in 1972 found age to be the major predictor of how well patients with CSM will do with any form of treatment. ${ }^{12,13}$ Nurick characterized CSM as a benign, nonprogressive condition, as had Lees and Turner, and recommended that surgical treatment be undertaken only in patients with progressive disability, which apparently rarely occurred. Phillips ${ }^{14}$ in 1973 , found that conservative treatment improved only a minority of the cases. None of these reports, however, used the same classification system of clinical disability, precluding meaningful comparison of results.

Because of the lack of prospective, randomized trials comparing surgical and non-surgical treatment of CSM, the decision about whether to operate is often based on the surgeon's own experience treating patients with CSM and on reports of results of surgical treatment. Previous investigations of the surgical treatment of cervical spine disease were limited to procedure-specific studies. ${ }^{1-16}$ Our study is population specific: it focuses on patients older than 70 years. Although comparison with other series was problematic, we used Nurick's criteria as modified by Lesoin et al. to evaluate our results ${ }^{9,12,13}$ because Nurick's scale is the scale most often cited in the literature, was readily applicable to our retrospective study and was estimated to be the least likely to be influenced by observer bias. Most of our patients (32/ 51) presented with Nurick's stage II disease. In 21 of these patients, CSM improved to stage I. In 10, the condition stabilized, and in one, the condition worsened to stage III (Table 5). Overall, $60.8 \%$ of our patients had improvement in their Nurick assessment, and $33.3 \%$ remained stable. Although physician or patient bias (eg 'placebo effect') might possibly have influenced these results, this is a recognized limitation of any retrospective study.

Some referring physicians have considered old age with its attendant infirmities, to be a relative contraindication to operative intervention. In a study by Saunders et al., in which approximately $25 \%$ of the patients were 70 years of age or older, cardiovascular conditions and osteoporosis were more important than age in determining outcome. Lunsford et al., however, reported poor results in patients aged 60 years or older, in their study of anterior surgery for CSM. This study demonstrates that a variety of surgical approaches for treatment of CSM may be undertaken in the symptomatic geriatric population with good efficacy and acceptable risks of morbidity or mortality. In addition to evaluating surgical outcome, one of the major goals of this study was to identify the medical and surgical complications reasonably to be anticipated in elderly patients. Cardiopulmonary disease was the major premorbid medical condition (39/51), Geriatric patients have decreased diastolic filling rates, decreased contractility, increased systemic vascular resistance and decreased pulmonary functional reserve.

Postoperatively, seven patients developed clinically detected atelectasis. The condition resolved with 
incentive spirometry (a triflow inhalation/exhalation device) within 3-5 days. Only one case of pulmonary edema was noted. Five patients had labile hypertension episodes, and three patients had arrhythmias; both conditions resolved with medication. One patient died of cardiac arrest of undetermined etiology; this was the only postsurgical death in the series. Virtually all patients 70 years of age or older are at risk for these post surgical complications. Over the past two decades, however, advances in critical-care monitoring and intensive care have enabled the neurosurgeon to manage post-surgical complications more effectively. Similarly, advances in anesthetic techniques have allowed neurosurgeons to undertake operations in the elderly with less risk. We had five of $51(9.8 \%)$ patients that needed additional hospital stay to address these complications.

We conclude that corrective procedures for clinically significant non-traumatic cervical myelopathy in the geriatric population may be performed with acceptable risk of morbidity and reasonable expectation for clinical improvement.

\section{References}

1 Bohlman HH. Cervical spondylosis with moderate to severe myelopathy: A report of 17 cases treated by Robinson anterior cervical diskectomy and fusion. Spine 1977; 2: $151-162$.

2 Clark CR. Cervical myelopathy: History and physical findings. Spine 1988; 13: $847-849$.
3 Clarke E, Robinson PK. Cervical myelopathy. A complication of cervical spondylosis. Brain 1956; 79: $483-497$.

4 Epstein JA. The surgical management of cervical spinal stenosis, spondylosis and myeloradiculopathy by means of the posterior approach. Spine 1988; 13: $864-869$.

5 Frager C. Management of cervical disc lesions and spondylosis by posterior approaches. Clin Neurosurg 1976; 24: 488 - 507.

6 Hakuda S, Mochizuki T, Ogata M. Operations for cervical spondylitic myelopathy. A comparison of the results of anterior and posterior procedures. J Bone Joint Surg 1985; 67B: 609-615.

7 Hirabayashi $\mathrm{K}$ et al. Operative results and postoperative progression of ossification among patients with ossification of cervical posterior longitudinal ligament. Spine 1981; 60: $354-$ 364.

8 Hirabayashi K, Satomi K. Operative procedure and results of expansive open-door laminoplasty. Spine 1988; 13: 370-376.

9 Lesoin F et al. Results of surgical treatment of radiculopathy caused by cervical arthrosis based on 1000 operations. Surg Neurol 1985; 23: 350-355.

10 Lees F, Turner JWA. Natural history and prognosis of cervical spondylosis. $\mathrm{Br}$ Med $J$ 1963; 2: $1607-1610$.

11 Lunsford LD, Bissonette DJ, Zorub DS. Anterior surgery for cervical disc disease. Part 2: Treatment of cervical spondylitic myelopathy in 32 cases. J Neurosurg 1980; 53: $12-19$.

12 Nurick S. The natural history and results of surgical treatment of the spinal cord disorder associated with cervical spondylosis. Brain 1972; 95: $101-108$.

13 Nurick S. The pathogenesis of the spinal cord disorder associated with cervical spondylosis. Brain 1972; 95: 87-100.

14 Phillips DG. Surgical treatment of myelopathy with cervical spondylosis. J Neurol Neurosurg Psychiatry 1973; 6: 879-884.

15 Saunders RI et al. Central corpectomy for cervical spondylitic myelopathy: consecutive series with long-term follow-up evaluation. J Neurosurg 1991; 74: $163-170$. 